\title{
Quebra da dormência de macieiras 'Daiane' pelo uso do tidiazurom
}

\author{
Breaking the of dormancy of 'Daiane' apples by using thidiazuron
}

\author{
Mariuccia Schlichting De Martin ${ }^{1 *}$, José Luiz Petri ${ }^{1}$, Cristhian Leonardo Fenilii ${ }^{2}$ Gentil Carneiro \\ Gabardo $^{2,3}$
}

Recebido em 20/01/2016 / Aceito em 11/11/2016

\section{RESUMO}

O objetivo deste trabalho foi avaliar o efeito de diferentes concentrações de tidiazurom (TDZ) combinados com óleo mineral (OM) ou nitrato de cálcio na fenologia, indução da brotação e produção de frutos em macieira 'Daiane'. O experimento foi conduzido no município de Caçador, SC, durante os ciclos 2013/2014 e 2014/2015. Os tratamentos avaliados foram: testemunha; TDZ $250 \mathrm{mg} \mathrm{L}^{-1}+\mathrm{OM}$ 3,5\%; TDZ $125 \mathrm{mg} \mathrm{L}^{-1}+$ OM 3,5\%; TDZ $250 \mathrm{mg}$ $\mathrm{L}^{-1}+$ nitrato de cálcio $5 \%$; cianamida hidrogenada (CH) $0,7 \%+$ OM 3,5\%. O delineamento experimental foi em blocos ao acaso, com seis repetições. A aplicação de indutores de brotação reduziu o período de florescimento na safra 2013/2014, ocasionando ainda maior brotação de gemas axilares em relação à testemunha, para as duas safras avaliadas. O TDZ + $\mathrm{OM}$ foi mais eficiente na brotação de gemas axilares comparativamente aos demais tratamentos. O TDZ $+\mathrm{OM}$ e a $\mathrm{CH}+\mathrm{OM}$ apresentaram efeito similar na brotação de gemas terminais, sendo superiores à aplicação de $\mathrm{TDZ}+$ nitrato de $\mathrm{Ca}$. No ciclo 2013/2014, a testemunha e os tratamentos TDZ 250 $\mathrm{mg} \mathrm{L^{-1 }}+\mathrm{OM}$ e $\mathrm{CH}+\mathrm{OM}$ apresentaram produção de frutos semelhantes, com maiores valores em comparação aos demais tratamentos. Para a safra 2014/2015, a massa média de frutos foi superior nas plantas submetidas às aplicações de TDZ $250 \mathrm{mg} \mathrm{L}^{-1}$ $+\mathrm{OM}$ e $\mathrm{CH}+\mathrm{OM}$. O TDZ, em combinação com o $\mathrm{OM}$, é eficiente na indução da brotação de macieiras 'Daiane', constituindo uma alternativa viável à aplicação de $\mathrm{CH}$.

PALAVRAS-CHAVE: Malus domestica, indutores de brotação, cianamida hidrogenada, óleo mineral, nitrato de cálcio.

\section{ABSTRACT}

The aim of this study was to evaluate the effect of different concentrations of thidiazuron (TDZ) combined with mineral oil (MO) or calcium nitrate on phenology, bud breaking and fruit production in 'Daiane' apple trees. The experiment was carried in Caçador, SC, during the 2013/2014 and 2014/2015 seasons. The treatments evaluated were control (without application); TDZ $250 \mathrm{mg} \mathrm{L}^{-1}+\mathrm{MO} 3,5 \%$; TDZ $125 \mathrm{mg} \mathrm{L}^{-1}+$ MO 3,5\%; TDZ $250 \mathrm{mg} \mathrm{L}^{-1}+$ calcium nitrate $5 \%$; hydrogen cyanamide (HC) $0,7 \%$ + MO 3,5\%. A randomized block design was used, with six repetitions. The application of bud break promoters reduced the flowering period in 2013/2014 season, leading to a higher bud break of axillary buds than control in the two seasons. TDZ + MO was more effective for bud break in axillary buds than the other treatments. TDZ $+\mathrm{MO}$ and $\mathrm{HC}+\mathrm{MO}$ had similar effect on bud break in terminal buds, with higher values than TDZ + calcium nitrate applications. In 2013/2014 season, the control and TDZ $250 \mathrm{mg} \mathrm{L}^{-1}$ $+\mathrm{MO}$ and $\mathrm{HC}+\mathrm{MO}$ treatments showed similar fruit production, being higher when compared to the other treatments. For 2014/2015, the fruit weight was higher in plants submitted to applications of TDZ $250 \mathrm{mg} \mathrm{L}^{-1}$ $+\mathrm{MO}$ and $\mathrm{HC}+\mathrm{MO}$. The TDZ combined with MO is efficient in bud break induction of 'Daiane' apples, being a viable alternative to $\mathrm{HC}$ application.

KEYWORDS: Malus domestica, bud break promoters, calcium nitrate, mineral oil, hydrogen cyanamide.

${ }^{1}$ Empresa de Pesquisa Agropecuária e Extensão Rural de Santa Catarina, Caçador, SC, Brasil.

${ }^{2}$ Universidade do Estado de Santa Catarina, Lages, SC, Brasil.

${ }^{3}$ Universidade Alto Vale do Rio do Peixe, Caçador, SC, Brasil.

*Autor para correspondência <mariucciamartin@epagri.sc.gov.br> 


\section{INTRODUÇÃO}

A macieira (Malus domestica Borkh.) caracteriza-se pela queda das folhas no final do ciclo e entrada em dormência durante o inverno. Essa inatividade fisiológica permite a sobrevivência de frutíferas de clima temperado em condições de baixas temperaturas, proporcionando condições naturais para que se inicie um novo ciclo vegetativo na primavera (OLIVEIRA et al. 2008). Para que ocorra a brotação e a floração da macieira de forma satisfatória, faz-se necessário que as plantas sejam expostas às baixas temperaturas durante o período de outono e de inverno em quantidade suficiente para atender suas necessidades fisiológicas (OLSEN 2006, FRANCESCATTO 2014).

Tanto a regularidade como a quantidade de frio são indispensáveis para a superação natural da dormência. Em condições de insuficiência em frio hibernal, a macieira apresenta anormalidades em relação à brotação, tendo repercussão durante o ciclo vegetativo e acarretando em redução tanto na produtividade quanto na qualidade dos frutos produzidos (HAWERROTH et al. 2010a, ANZANELLO et al. 2014). No Brasil, a maior parte da cultura da macieira está localizada em áreas onde a exigência em frio das principais cultivares não é plenamente satisfeita (RUFATO et al. 2010, PETRI et al. 2011).

'Daiane' é uma cultivar de macieira, resultante do cruzamento entre 'Gala' ( + ) e 'Princesa' ( $\left.)^{\Uparrow}\right)$, que produz frutos semelhantes à 'Gala' quanto à aparência, sabor e aroma (DENARDI \& CAMILO 1998). Dentre as vantagens que essa cultivar apresenta, destacam-se a resistência à mancha foliar de glomerella e período da colheita comercial concentrado entre o da 'Gala' e da 'Fuji', as quais representam mais de $90 \%$ da produção nacional, favorecendo o escalonamento da colheita (STANGER et al. 2013). Todavia, assim como essas cultivares, a 'Daiane' também não tem sua necessidade de frio hibernal totalmente satisfeita, uma vez que apresenta um requerimento de 1300 a 1600 unidades de frio (UF), o que a classifica como uma cultivar de média a alta exigência (EPAGRI 2013).

Em condições de invernos mais amenos, práticas como a utilização de produtos químicos para a superação artificial da dormência têm sido utilizadas com o objetivo de amenizar alguns dos problemas decorrentes da deficiência de frio, proporcionando brotação e floração adequadas (PETRI et al. 2006, RUFATO et al. 2010, FRANCESCATTO 2014). Atualmente, dentre as opções disponíveis no mercado, a cianamida hidrogenada $(\mathrm{CH})$ é a principal substância utilizada comercialmente na indução da brotação de várias espécies frutíferas (HAWERROTH et al. 2009, 2010a). Segundo PETRI et al. (2011), o uso da $\mathrm{CH}$ combinado ao óleo mineral (OM), além de proporcionar economia ao produtor, propicia aumento na produtividade, constituindo a principal estratégia utilizada para indução de brotação em macieiras. Contudo, o uso exclusivo da $\mathrm{CH}$ como indutor de brotação em macieira apresenta alguns aspectos limitantes, como a alta toxicidade para o aplicador (HAWERROTH et al. 2010a). Nesse sentido, estudos têm sido realizados com produtos alternativos para a superação de dormência de plantas frutíferas de clima temperado (HAWERROTH et al. 2010b, PETRI et al. 2014).

O tidiazurom (TDZ) é uma feniluréia que apresenta atividade similar à da citocinina (GUO et al. 2011), um dos principais fitormônios envolvidos no processo de quebra de dormência em frutíferas de clima temperado (JANA 2015). De acordo com PETRI et al. (2006), o TDZ em combinação com o OM, apresenta forte efeito na brotação de gemas axilares de maçãs 'Gala' e 'Golden Delicious', inclusive nas gemas de base e em ramos envelhecidos da planta. Segundo o Ministério da Agricultura, esse composto está inserido dentro da classe toxicológica IV (pouco tóxica), representando menor risco à saúde humana em relação à $\mathrm{CH}$, a qual se enquadra dentro da classe toxicológica I (extremamente tóxica).

Além do efeito do OM, o uso do nitrato de cálcio associado a outros indutores tem se mostrado promissor na indução de brotação em gemas de macieira (HAWERROTH et al. 2010b, PETRI et al. 2014). Porém, o resultado da sua aplicação em combinação com o TDZ precisa ser melhor elucidado.

Este trabalho teve como objetivo avaliar o efeito de diferentes concentrações de tidiazurom combinadas com óleo mineral ou nitrato de cálcio na indução da brotação e na produção de frutos em macieira 'Daiane'.

\section{MATERIAL E MÉTODOS}

O experimento foi conduzido no município de Caçador, SC (situado 26 $42^{\prime} 32^{\prime \prime S}$ de latitude, $51^{\circ} 00^{\prime} 50^{\prime \prime} \mathrm{W}$ de longitude e $960 \mathrm{~m}$ de altitude) durante 
os ciclos 2013/2014 e 2014/2015. O clima dessa região caracteriza-se como temperado constantemente úmido, com verão ameno, segundo classificação climática de Köppen, do tipo "Cfb".

Foram utilizadas macieiras da cultivar Daiane com 12 anos de idade, enxertadas sobre o portaenxerto M-7, conduzidas no sistema de líder central e espaçadas em $2 \mathrm{~m}$ na linha e $5 \mathrm{~m}$ entre linhas. O pomar utilizado foi conduzido de acordo com as práticas de manejo recomendadas para o sistema de produção da macieira.

$\mathrm{O}$ delineamento experimental foi em blocos ao acaso, com seis repetições, sendo cada unidade experimental composta por uma planta. Os tratamentos avaliados foram: 1) testemunha (sem aplicação); 2) tidiazurom (TDZ) $250 \mathrm{mg} \mathrm{L}^{-1}+$ óleo mineral (OM) 3,5\%; 3) TDZ $125 \mathrm{mg} \mathrm{L}^{-1}+$ OM 3,5\%; 4) TDZ $250 \mathrm{mg} \mathrm{L}^{-1}+$ nitrato de cálcio $\left(\mathrm{Ca}\left(\mathrm{NO}_{3}\right)_{2}\right) 5 \%$; 5) cianamida hidrogenada $(\mathrm{CH}) 0,7 \%+\mathrm{OM} \mathrm{3,5 \%}$.

Como fonte de TDZ, OM, nitrato de cálcio e $\mathrm{CH}$ foram utilizados os produtos comerciais Dropp ${ }^{\circledR}$, Assist $^{\circledR}$, Nitroactive ${ }^{\circledR}$ e Dormex ${ }^{\circledR}$, respectivamente. A aplicação dos tratamentos foi efetuada através de aspersão com pulverizador costal motorizado até próximo do ponto de gotejamento, utilizando-se volume médio de calda equivalente a $1000 \mathrm{~L} \mathrm{ha}^{-1}$. Cada tratamento foi aplicado nas mesmas plantas durante os dois ciclos de produção avaliados.

Nos ciclos 2013/2014 e 2014/2015, as aplicações dos tratamentos foram realizadas em 11/09/13 e 04/09/2014, respectivamente. No momento da aplicação, todas as gemas estavam dormentes no estádio B (IUCHI 2006).

Foram obtidas as temperaturas mensais máximas, mínimas e médias entre os meses de Agosto e Abril, para as safras 2013/2014 e 2014/2015 (Tabela 1). Os dados meteorológicos utilizados neste trabalho provêm de uma estação meteorológica situada na Estação experimental da EPAGRI de Caçador, SC (EPAGRI/CIRAM 2015).

O acúmulo de frio durante o período hibernal até a data de aplicação dos tratamentos foi de 940 e 884 unidades de frio (UF) (EPAGRI/CIRAM 2015), segundo o modelo Carolina do Norte Modificado, para os ciclos 2013/2014 e 2014/2015, respectivamente.

Para ambos os anos, foi avaliada a fenologia durante o período da floração, os percentuais de brotação de gemas axilares e de gemas terminais, a produção de frutos por planta e a massa média dos frutos. A avaliação da fenologia do florescimento consistiu na determinação das datas de ocorrência das fases de início, plena e final de floração para cada tratamento. O início de floração foi considerado quando as plantas estavam com $10 \%$ de flores abertas, a plena floração quando verificado mais de $70 \%$ de flores abertas e o fim de floração foi dado quando as últimas flores estavam abertas.

As brotações de gemas axilares e terminais foram estimadas aos 30 e 60 dias após a quebra de dormência (DAQD), para os dois anos de avaliação. Para tanto, foram amostradas cinco brindilas de ano por planta, localizadas no terço médio da planta,

Tabela 1 - Temperaturas mensais máximas, mínimas e médias no município de Caçador, SC, durante os ciclos 2013/2014 e 2014/2015.

Table 1 - Maximum, minimum and average monthly temperature in Caçador, SC, during 2013/2014 and 2014/2015 seasons.

\begin{tabular}{lcccccc}
\hline & \multicolumn{5}{c}{ Temperatura $\left({ }^{\circ} \mathrm{C}\right)$} \\
\cline { 2 - 7 } Mês & \multicolumn{7}{c}{ Safra 2013/2014 } & \multicolumn{3}{c}{ Safra 2014/2015 } \\
\cline { 2 - 7 } & Máxima & Mínima & Média & Máxima & Mínima & Média \\
\hline Abril & 22,9 & 10,4 & 15,4 & 22,8 & 13,9 & 17,3 \\
Maio & 19,9 & 9,3 & 13,6 & 19,5 & 9,8 & 13,7 \\
Junho & 18,1 & 8,9 & 12,6 & 17,9 & 9,9 & 13,0 \\
Julho & 18,2 & 6,2 & 11,0 & 17,8 & 8,3 & 12,2 \\
Agosto & 19,0 & 7,6 & 12,2 & 22,0 & 7,7 & 13,6 \\
Setembro & 20,5 & 10,3 & 14,4 & 21,7 & 11,9 & 16,0 \\
Outubro & 23,1 & 12,1 & 16,7 & 25,5 & 13,4 & 18,6 \\
Novembro & 24,5 & 14,3 & 18,8 & 24,7 & 14,3 & 18,8 \\
Dezembro & 27,3 & 15,3 & 20,6 & 24,9 & 15,8 & 20,1 \\
Janeiro & 28,2 & 16,5 & 21,3 & 27,2 & 16,7 & 21,2 \\
Fevereiro & 28,5 & 16,1 & 21,1 & 26,2 & 16,1 & 20,2 \\
Março & 25,0 & 14,8 & 18,8 & 26,0 & 15,7 & 19,6 \\
\hline
\end{tabular}


contabilizando-se o número de gemas axilares brotadas e não brotadas. Uma ramificação lateral de cada planta foi previamente selecionada, para estimar o percentual de brotação de gemas terminais (HAWERROTH et al. 2010a).

A determinação da produção por planta e massa média dos frutos foi obtida mediante a colheita da totalidade dos frutos produzidos por cada planta. Após a colheita, os frutos foram pesados e contabilizados. A produção foi expressa em kg planta ${ }^{-1} \mathrm{e}$ frutos planta $^{-1} \mathrm{e}$ a massa média dos frutos em $\mathrm{g}$.

Os dados de brotação de gemas axilares e terminais foram transformados através da equação arco seno $(\mathrm{x} / 100)^{1 / 2}$. Dados de produção, expressos em número de frutos por planta, foram transformados pela fórmula $(\mathrm{x}+1)^{1 / 2}$. Para a análise estatística, os dados foram submetidos à análise de variância (ANOVA), e as médias dos tratamentos comparadas pelo teste de Scott-Knott $(p \leq 0,05)$. Para estes procedimentos foi utilizado o programa estatístico Sisvar 5.3 (FERREIRA 2008).

\section{RESULTADOS E DISCUSSÃO}

Para a safra 2013/2014, o estádio fenológico C-C3 ocorreu quatro dias mais tarde na testemunha em relação às plantas tratadas com indutores de brotação (Tabela 2). O início da brotação também ocorreu com dois dias de antecedência nas plantas tratadas em relação àquelas que não receberam tratamento. As aplicações do TDZ $250 \mathrm{mg} \mathrm{L}^{-1}+\mathrm{OM}$ e TDZ $250 \mathrm{mg} \mathrm{L}^{-1}+$ nitrato de cálcio reduziram o período de florescimento em 11 dias em comparação à testemunha, e em três dias quando comparadas às aplicações de TDZ $125 \mathrm{mg} \mathrm{L}^{-1}+\mathrm{OM} \mathrm{e} \mathrm{CH}+\mathrm{OM}$.

No ciclo 2014/2015, a aplicação dos indutores de brotação adiantou a floração em comparação à testemunha (Tabela 2). As plantas submetidas à aplicação de TDZ + OM tiveram um período de florescimento nove e dez dias menor em comparação à testemunha, para as doses de 250 e $125 \mathrm{mg} \mathrm{L}^{-1}$, respectivamente. Já os tratamentos TDZ $250 \mathrm{mg} \mathrm{L}^{-1}+$ nitrato de cálcio e $\mathrm{CH}+\mathrm{OM}$ reduziram o período de florescimento em sete e cinco dias, respectivamente, em relação à testemunha. Esse efeito dos indutores de brotação na redução do período de florescimento pode ser desejado, uma vez que o prolongamento do mesmo pode dificultar a realização de algumas práticas culturais, como raleio e controle de doenças, devido à ocorrência de diferentes estádios fenológicos dentro de uma mesma planta (PETRI \& LEITE 2004, HAWERROTH et al. 2010b). Por outro lado, a concentração do período de florescimento pode reduzir a frutificação efetiva, devido a fatores como a não coincidência do período de florescimento com o das plantas polinizadoras, baixa densidade de insetos polinizadores e mesmo a ocorrência de condições climáticas adversas durante o período de polinização (HAWERROTH et al. 2010a).

Não apenas a quantidade, mas também a qualidade e a regularidade do frio influenciam o processo de superação da dormência (ANZANELLO et al. 2014). Assim, ainda que os anos de 2013 e 2014 tenham apresentado unidades de frio semelhantes ( 940 e 884 UF, respectivamente), a variação da temperatura observada entre as duas safras, especialmente durante o inverno (Tabela 1) pode ter sido a causa mais provável das diferenças no período de florescimento, bem como na eficiência da quebra de dormência com produtos químicos, que ocorreu de um ano para outro.

Para as duas safras estudadas, os tratamentos com indutores proporcionaram maior brotação de gemas axilares em relação à testemunha (Tabela 3). Possivelmente, os baixos valores encontrados na testemunha tenham ocorrido devido à quantidade insuficiente de frio hibernal para superação natural da dormência nos dois anos avaliados, justificando o uso de indutores de brotação. Regiões com baixo acúmulo de frio estimulam o crescimento terminal de frutíferas de clima temperado, inibindo a brotação das gemas axilares (PETRI et al. 2006, FRANCESCATTO 2014).

No ano agrícola 2013/2014, os tratamentos com TDZ + OM foram mais eficientes para a brotação de gemas axilares em comparação aos demais tratamentos, tanto aos 30 quanto aos 60 DAQD (Tabela 3). No ciclo 2014/2015, a brotação de gemas axilares de plantas tratadas com 250 e $125 \mathrm{mg} \mathrm{L}^{-1} \mathrm{de}$ $\mathrm{TDZ}+\mathrm{OM}$ foi similar a de plantas tratadas com CH + OM aos 30 DAQD, sendo, contudo, superior em relação à brotação de plantas tratadas com TDZ + nitrato de cálcio. Já na avaliação realizada 60 DAQD, as plantas tratadas com $\mathrm{CH}+\mathrm{OM}$ apresentaram menor brotação de gemas axilares em comparação àquelas tratadas com as duas concentrações de TDZ + OM. É importante salientar que a falta de brotação das gemas axilares antecipa a brotação de gemas terminais, estabelecendo forte dominância apical, comprometendo a formação de novos órgãos reprodutivos e aumentando demasiadamente $\mathrm{o}$ 
Tabela 2 - Datas médias correspondentes aos estádios fenológicos C-C3, início da brotação e floração de macieiras 'Daiane' tratadas com diferentes indutores de brotação durante os ciclos 2013/2014 e 2014/2015. Caçador, SC.

Table 2 - Average dates corresponding to phenological stages C-C3, beginning of bud break and flowering in 'Daiane' apple trees treated with different bud break promoters during 2013/2014 and 2014/2015 seasons. Caçador, SC.

\begin{tabular}{|c|c|c|c|c|c|}
\hline \multirow{2}{*}{ Tratamento } & \multirow{2}{*}{$\mathrm{C}-\mathrm{C} 3$} & \multirow{2}{*}{$\begin{array}{l}\text { Início da } \\
\text { brotação }\end{array}$} & \multicolumn{3}{|c|}{ Floração } \\
\hline & & & Início & Plena & Final \\
\hline & \multicolumn{5}{|c|}{ Ciclo 2013/2014 } \\
\hline Testemunha & $14 /$ out & $14 /$ out & 14/out & $25 /$ out & $05 /$ nov \\
\hline TDZ $250 \mathrm{mg} \mathrm{L}^{-1}+$ OM 3,5\% & 10/out & $12 /$ out & $14 /$ out & 21/out & $25 /$ out \\
\hline TDZ $125 \mathrm{mg} \mathrm{L}^{-1}+$ OM 3,5\% & $10 /$ out & $12 /$ out & 14/out & 21/out & $28 /$ out \\
\hline $\mathrm{TDZ} 250 \mathrm{mg} \mathrm{L}^{-1}+\mathrm{Ca}\left(\mathrm{NO}_{3}\right)_{2} 5 \%$ & $10 /$ out & $12 /$ out & 14/out & $22 /$ out & $25 /$ out \\
\hline \multirow[t]{2}{*}{$\mathrm{CH} 0,7 \%+\mathrm{OM} 3,5 \%$} & 10/out & $12 /$ out & $14 /$ out & 21/out & 28/out \\
\hline & \multicolumn{5}{|c|}{ Ciclo 2014/2015 } \\
\hline Testemunha & $22 /$ set & $26 /$ set & $10 /$ out & $25 /$ out & 30/out \\
\hline TDZ $250 \mathrm{mg} \mathrm{L}^{-1}+$ OM 3,5\% & $23 /$ set & $27 /$ set & 02/out & 08/out & $13 /$ out \\
\hline TDZ $125 \mathrm{mg} \mathrm{L}^{-1}+$ OM 3,5\% & $30 /$ set & 03/out & 03/out & 08/out & $13 /$ out \\
\hline $\mathrm{TDZ} 250 \mathrm{mg} \mathrm{L}^{-1}+\mathrm{Ca}\left(\mathrm{NO}_{3}\right)_{2} 5 \%$ & $24 /$ set & $29 /$ set & $30 /$ set & 08/out & $13 /$ out \\
\hline $\mathrm{CH} 0,7 \%+\mathrm{OM} 3,5 \%$ & $23 /$ set & $26 /$ set & $28 /$ set & 08/out & $13 /$ out \\
\hline
\end{tabular}

Estádio fenológico $\mathrm{C}=$ pontas verdes; Estádio fenológico $\mathrm{C} 3$ = meia polegada verde; TDZ = tidiazurom; $\mathrm{OM}=$ óleo mineral; $\mathrm{CH}=$ cianamida hidrogenada.

Tabela 3 - Brotação de gemas axilares e terminais aos 30 e 60 dias após a quebra de dormência (DAQD) de macieiras 'Daiane' tratadas com diferentes indutores de brotação durante os ciclos 2013/2014 e 2014/2015. Caçador, SC.

Table 3 - Bud break of axillary and terminal buds 30 and 60 days after breaking the dormancy of 'Daiane' apple trees treated with different bud break promoters during 2013/2014 and 2014/2015 seasons. Caçador, SC.

\begin{tabular}{|c|c|c|c|c|}
\hline \multirow{3}{*}{ Tratamento } & \multicolumn{4}{|c|}{ Brotação de gemas (\%) } \\
\hline & \multicolumn{2}{|c|}{ Axilares } & \multicolumn{2}{|c|}{ Terminais } \\
\hline & $30 \mathrm{DAQD}$ & $60 \mathrm{DAQD}$ & $30 \mathrm{DAQD}$ & 60 DAQD \\
\hline & \multicolumn{4}{|c|}{ Ciclo 2013/2014 } \\
\hline Testemunha & $4,0 \mathrm{c}$ & $9,2 \mathrm{c}$ & $64,1 \mathrm{~b}$ & $75,5 \mathrm{~b}$ \\
\hline TDZ $250 \mathrm{mg} \mathrm{L}^{-1}+$ OM 3,5\% & $70,9 \mathrm{a}$ & $75,1 \mathrm{a}$ & $85,0 \mathrm{a}$ & $92,1 \mathrm{a}$ \\
\hline TDZ $125 \mathrm{mg} \mathrm{L}^{-1}+$ OM 3,5\% & $54,6 \mathrm{a}$ & $57,1 \mathrm{a}$ & $87,7 \mathrm{a}$ & $92,4 \mathrm{a}$ \\
\hline $\mathrm{TDZ} 250 \mathrm{mg} \mathrm{L}^{-1}+\mathrm{Ca}\left(\mathrm{NO}_{3}\right)_{2} 5 \%$ & $18,2 \mathrm{~b}$ & $30,4 \mathrm{~b}$ & $81,7 \mathrm{~b}$ & $88,4 \mathrm{~b}$ \\
\hline $\mathrm{CH} 0,7 \%+\mathrm{OM} 3,5 \%$ & $19,7 \mathrm{~b}$ & $21,5 \mathrm{~b}$ & 95,9 a & $98,0 \mathrm{a}$ \\
\hline \multirow[t]{2}{*}{$\mathrm{CV}(\%)$} & 29,6 & 31,2 & 15,7 & 12,9 \\
\hline & \multicolumn{4}{|c|}{ Ciclo 2014/2015 } \\
\hline Testemunha & $14,0 \mathrm{c}$ & $25,7 \mathrm{c}$ & $41,5 \mathrm{c}$ & $89,0 \mathrm{~b}$ \\
\hline TDZ $250 \mathrm{mg} \mathrm{L}^{-1}+\mathrm{OM} 3,5 \%$ & 57,9 a & $62,5 \mathrm{a}$ & 94,5 a & $100,0 \mathrm{a}$ \\
\hline TDZ $125 \mathrm{mg} \mathrm{L}^{-1}+$ OM 3,5\% & 64,6 a & $71,1 \mathrm{a}$ & 95,6 a & 99,6 a \\
\hline $\mathrm{TDZ} 250 \mathrm{mg} \mathrm{L}^{-1}+\mathrm{Ca}\left(\mathrm{NO}_{3}\right)_{2} 5 \%$ & $30,2 \mathrm{~b}$ & $43,2 \mathrm{~b}$ & $82,0 \mathrm{~b}$ & $98,1 \mathrm{a}$ \\
\hline $\mathrm{CH} 0,7 \%+\mathrm{OM} 3,5 \%$ & $43,0 \mathrm{a}$ & $49,3 \mathrm{~b}$ & $100,0 \mathrm{a}$ & $100,0 \mathrm{a}$ \\
\hline $\mathrm{CV}(\%)$ & 26,2 & 17,4 & 17,2 & 7,3 \\
\hline
\end{tabular}

$\mathrm{TDZ}=$ tidiazurom; $\mathrm{OM}=$ óleo mineral; $\mathrm{CH}=$ cianamida hidrogenada.

Médias seguidas pela mesma letra, na vertical, não diferem entre si pelo teste de Tukey $(\mathrm{p}<0,05)$. 
desenvolvimento vegetativo (PETRI et al. 2006, FRANCESCATTO 2014).

Para o ano agrícola 2013/2014, a brotação de gemas terminais foi mais elevada em plantas submetidas aos tratamentos com $\mathrm{TDZ}+\mathrm{OM}$ e $\mathrm{CH}$ + OM comparativamente às demais, tanto aos 30 quanto aos 60 DAQD (Tabela 3). Para o ano agrícola 2014/2015, nos 30 DAQD, o menor número de gemas terminais brotadas foi obtido pela testemunha. Os tratamentos $\mathrm{TDZ}+\mathrm{OM}$ e $\mathrm{CH}+\mathrm{OM}$ apresentaram efeito similar na brotação, sendo superiores ao tratamento $\mathrm{TDZ}+$ nitrato de $\mathrm{Ca}$. $\mathrm{Na}$ avaliação realizada $60 \mathrm{DAQD}$, todos os indutores de brotação proporcionaram um aumento similar da brotação de gemas terminais em comparação à testemunha.

Ainda que a aplicação de indutores tenha ocasionado maior brotação de gemas terminais, foi possível perceber que esses percentuais também foram elevados na testemunha aos 60 DAQD, apresentando valores superiores a $75 \%$ em ambos os anos (Tabela 3). Em estudo conduzido com maçãs 'Royal Gala' durante dois anos de avaliação, HAWERROTH et al. (2010a) observaram que o uso de agentes químicos na indução da brotação de gemas axilares é mais importante que em gemas terminais, visto que mesmo em condições de não suprimento do requerimento em frio, a brotação das gemas terminais é elevada, o que não ocorre para brotação de gemas axilares.

Em estudo conduzido com macieiras 'Imperial Gala' e 'Fuji Suprema', HAWERROTH et al. (2010b) verificaram que a combinação Erger $^{\circledR}+$ nitrato de $\mathrm{Ca}$ apresentou desempenho similar ao da $\mathrm{CH}+\mathrm{OM}$ na brotação de gemas e na uniformização da brotação, se mostrando uma alternativa eficiente na indução da brotação em macieiras. Todavia, no presente trabalho, a combinação de TDZ com nitrato de Ca se mostrou menos eficiente na brotação de gemas axilares e terminais quando comparada aos tratamentos TDZ + OM, em ambos os anos de avaliação.

A testemunha e os tratamentos TDZ $250 \mathrm{mg} \mathrm{L}^{-1}+\mathrm{OM}$ e $\mathrm{CH}+\mathrm{OM}$ apresentaram efeito similar na produção de frutos por planta, com maior produção em comparação aos demais tratamentos para o ciclo 2013/2014 (Tabela 4). Para o ciclo 2014/2015, não houve efeito da aplicação dos indutores de brotação sobre a produção de frutos. Ainda que a aplicação dos indutores de brotação não tenha ocasionado maior produção em comparação à testemunha, para os

Tabela 4 - Produção e massa média de frutos de macieiras 'Daiane' tratadas com diferentes indutores de brotação durante os ciclos 2013/2014 e 2014/2015. Caçador, SC.

Table 4 - Fruit production and weight of 'Daiane' apple trees treated with different bud break promoters during 2013/2014 and 2014/2015 seasons. Caçador, SC.

\begin{tabular}{|c|c|c|c|}
\hline \multirow{2}{*}{ Tratamento } & \multicolumn{2}{|c|}{ Produção de frutos } & \multirow{2}{*}{$\begin{array}{l}\text { Massa média dos frutos } \\
(\mathrm{g})\end{array}$} \\
\hline & $\mathrm{kg}_{\text {planta }}{ }^{-1}$ & frutos planta ${ }^{-1}$ & \\
\hline \multicolumn{4}{|c|}{ Ciclo 2013/2014 } \\
\hline Testemunha & $23,2 \mathrm{a}$ & $146,7 \mathrm{a}$ & $160,0^{\mathrm{ns}}$ \\
\hline TDZ $250 \mathrm{mg} \mathrm{L}^{-1}+\mathrm{OM} 3,5 \%$ & $22,0 \mathrm{a}$ & $134,3 \mathrm{a}$ & 164,1 \\
\hline $\mathrm{TDZ} 125 \mathrm{mg} \mathrm{L}^{-1}+\mathrm{OM} 3,5 \%$ & $13,8 b$ & $86,8 \mathrm{~b}$ & 160,6 \\
\hline $\mathrm{TDZ} 250 \mathrm{mg} \mathrm{L}^{-1}+\mathrm{Ca}\left(\mathrm{NO}_{3}\right)_{2} 5 \%$ & $7,9 b$ & $49,2 \mathrm{~b}$ & 160,8 \\
\hline $\mathrm{CH} 0,7 \%+\mathrm{OM} 3,5 \%$ & $23,4 a$ & $136,7 \mathrm{a}$ & 173,1 \\
\hline CV $(\%)$ & 39,2 & 22,0 & 5,8 \\
\hline \multicolumn{4}{|c|}{ Ciclo 2014/2015 } \\
\hline Testemunha & $11,4^{\mathrm{ns}}$ & $96,3^{\mathrm{ns}}$ & $117,7 \mathrm{~b}$ \\
\hline TDZ $250 \mathrm{mg} \mathrm{L}^{-1}+\mathrm{OM} 3,5 \%$ & 16,6 & 127 & $132,2 \mathrm{a}$ \\
\hline TDZ $125 \mathrm{mg} \mathrm{L}^{-1}+$ OM 3,5\% & 7,5 & 59,5 & $125,5 \mathrm{~b}$ \\
\hline $\mathrm{TDZ} 250 \mathrm{mg} \mathrm{L}^{-1}+\mathrm{Ca}\left(\mathrm{NO}_{3}\right)_{2} 5 \%$ & 8,2 & 71,3 & $117,5 \mathrm{~b}$ \\
\hline $\mathrm{CH} 0,7 \%+\mathrm{OM} 3,5 \%$ & 12,3 & 88,8 & $139,3 \mathrm{a}$ \\
\hline $\mathrm{CV}(\%)$ & 47,7 & 24,5 & 7,7 \\
\hline
\end{tabular}

$\mathrm{TDZ}=$ tidiazurom; $\mathrm{OM}=$ óleo mineral; $\mathrm{CH}=$ cianamida hidrogenada.

Médias seguidas pela mesma letra, na vertical, não diferem entre si pelo teste de Tukey $(\mathrm{p}<0,05)$; ${ }^{\text {ns: não }}$ significativo $(\mathrm{p}>0,05)$. 
dois anos de avaliação, o efeito dos indutores sobre a brotação das gemas axilares propicia um aumento no número de esporões e consequente aumento na quantidade de cachos florais, o que se intensifica com o decorrer dos anos (PETRI et al. 2006).

A massa média de frutos não apresentou diferenças entre tratamentos na safra 2013/2014 (Tabela4). Contudo, nociclo2014/2015, os tratamentos TDZ $250 \mathrm{mg} \mathrm{L}^{-1}+\mathrm{OM}$ e $\mathrm{CH}+\mathrm{OM}$ propiciaram aos frutos uma maior massa média em comparação aos demais. Para o presente trabalho, a maior massa dos frutos desses tratamentos pode ter ocorrido devido ao aumento da brotação de gemas axilares e terminais e, consequentemente, a um incremento na capacidade de produção de fotoassimilados devido ao maior desenvolvimento foliar (HAWERROTH et al. 2010a).

\section{CONCLUSÃO}

O TDZ nas doses de 125 e $250 \mathrm{mg} \mathrm{L}^{-1}$, quando utilizado em conjunto com o OM 3,5\%, é uma alternativa viável na quebra de dormência de macieiras 'Daiane', proporcionando brotação de gemas similar ou até mesmo superior à do tratamento padrão $(\mathrm{CH}$ $0,7 \%+$ OM 3,5\%).

$\mathrm{Na}$ dose de $250 \mathrm{mg} \mathrm{L}^{-1}$, o TDZ, combinado com o OM 3,5\%, não compromete a produção de macieiras 'Daiane', similarmente à aplicação de $\mathrm{CH}$ $0,7 \%+$ OM $3,5 \%$.

O TDZ combinado ao OM 3,5\% é mais eficiente na indução de brotação e na produtividade de maçãs 'Daiane' em comparação ao TDZ combinado ao nitrato de cálcio $5 \%$.

\section{REFERÊNCIAS}

ANZANELLO R et al. 2014. Bud dormancy in apple trees after thermal fluctuations. Pesquisa Agropecuária Brasileira 49: 457-464.

DENARDI F \& CAMILO AP. 1998. Daiane: Nova cultivar de macieira para colheita em março. Revista Agropecuária Catarinense 11: 6-8.

EPAGRI. Empresa de Pesquisa Agropecuária e Extensão Rural de Santa Catarina. 2013. Catálogo de cultivares 2013/2014. Florianópolis: Epagri. 38p.

EPAGRI/CIRAM - Empresa de Pesquisa Agropecuária e Extensão Rural de Santa Catarina/Centro de Informações de Recursos Ambientais e de Hidrometereologia. 2015. Monitoramento do frio. Florianópolis: Epagri. Disponível em: http://ciram.epagri.sc.gov.br. Acesso em: 01 out. 2015. FERREIRA DF. 2008. SISVAR: um programa para análises estatísticas e ensino de estatística. Revista Symposium 6:
36-41.

FRANCESCATTO P. 2014. Desenvolvimento das estruturas reprodutivas da macieira (Malus domestica Borkh.) sob diferentes condições climáticas: da formação da gema à colheita dos frutos. Tese (Doutorado em Recursos Genéticos Vegetais). Florianópolis: UFSC. 293p.

GUO B et al. 2011. Thidiazuron: A multi-dimensional plant growth regulator. African Journal of Biotechnology 10: 8984-9000.

HAWERROTH FJ et al. 2009. Fenologia, brotação de gemas e produção de frutos de macieira em resposta à aplicação de cianamida hidrogenada e óleo mineral. Bragantia 68: 961-971.

HAWERROTH FJ et al. 2010a. Cianamida hidrogenada, óleos mineral e vegetal na brotação de gemas e produção de macieiras 'Royal Gala'. Semina: Ciências Agrárias 31: 1145-1154.

HAWERROTH FJ et al. 2010b. Brotação de gemas em macieiras 'Imperial Gala' e 'Fuji Suprema' pelo uso de Erger $^{\circledR}$ e Nitrato de Cálcio. Revista Brasileira de Fruticultura 32: 343-350.

IUCHI VL. 2006. Botânica e fisiologia. In: EPAGRI. A Cultura da macieira. Florianópolis: Epagri. p.59-104.

JANA BR. 2015. Induction of flowering in asian pear (Pyrus spp.) - A review. International Journal of Plant Research 5: 57-63.

OLIVEIRA OR et al. 2008. Quebra de dormência de pereira 'Hosui' com uso de óleo mineral em dois tipos de condução. Revista Brasileira de Fruticultura 30: 409-413.

OLSEN JE. 2006. Mechanisms of dormancy regulation. Acta Horticulturae 727: 157-166.

PETRI JL \& LEITE GB. 2004. Consequences of insufficient winter chilling on apple tree bud-break. Acta Horticulturae 662: 53-60.

PETRI JL et al. 2011. Avanços na cultura da macieira no Brasil. Revista Brasileira de Fruticultura 33: 48-56.

PETRI JL et al. 2014. Chemical induction of budbreak: new generation products to replace hydrogen cyanamide. Acta Horticulturae 1042: 159-166.

PETRI JL et al. 2006. Dormência e indução a brotação em macieira. In: EPAGRI. A cultura da macieira. Florianópolis: Epagri. p.261-297.

RUFATO L et al. 2010. Bud break in different cultivars of apple trees in two regions of Santa Catarina State, Brazil. Acta Horticulturae 884: 643-646.

STANGER MC et al. 2013. Estádio de maturação para o período ideal de colheita de maçãs 'Daiane' destinadas à armazenagem. Revista Brasileira de Fruticultura 35: 977989. 